\title{
On the Effect of Imperfect Cophasing in MRC and EGC Receivers Over Correlated Weibull Fading
}

\author{
Bojana Z. Nikolic • Goran T. Djordjevic • \\ George K. Karagiannidis
}

Published online: 19 May 2010

C) Springer Science+Business Media, LLC. 2010

\begin{abstract}
This paper presents a comparative analysis of dual-branch maximal-ratio combining (MRC) and equal-gain combining (EGC) receivers with coherent modulations over correlated Weibull fading channels. The numerical and simulations results show the influence of imperfect cophasing, branch unbalancing and correlation on the error performance. It is interestingly shown that EGC has lower irreducible error floor than MRC in the presence of incoherent combining, while the higher value of the correlation coefficient results to lower irreducible error floor. Furthermore, the unbalance parameter has practically no influence on the irreducible error floor.
\end{abstract}

Keywords Bit error rate $\cdot$ Diversity $\cdot$ Fading channels $\cdot$ Simulations $\cdot$ Weibull distribution

\section{Introduction}

Weibull distribution has been used for modeling the multipath fading signal envelope in wireless communications, since it gives very good fit with measurement results both for indoor and outdoor environments [1,2]. Different diversity combining techniques are utilized in order to combat the influence of multipath fading on signal detection as maximal-ratio combining (MRC) and equal-gain combining (EGC). Both of them require cophasing at all receiver branches in order to eliminate the random signal phase fluctuations occurring during

B. Z. Nikolic · G. T. Djordjevic $(\varangle)$

Faculty of Electronic Engineering, Department of Telecommunications, Aleksandra Medvedeva 14, 18000 Nis, Serbia

e-mail: goran@elfak.ni.ac.rs

B. Z. Nikolic

e-mail: bojana.nikolic@elfak.ni.ac.rs

G. K. Karagiannidis

Department of Electrical \& Computer Engineering, Aristotle University of Thessaloniki,

54124 Thessaloniki, Greece

e-mail: geokarag@auth.gr 
transmission. The MRC also requires the estimation of the channel fading envelopes at all branches. It is well known that under the perfect cophasing, MRC is the optimal combining technique, while EGC is a suboptimal one. The so-called moment generation function (MGF) method was applied in [3] to study the average signal-to-noise ratio (SNR), the outage probability and the average symbol error rate for single and independent multibranch diversity reception over flat Weibull fading.

When receiver antennas are not sufficiently separated due to the small size mobile terminals, the fading envelopes at different branches are correlated [4, pp. 316-333, 5]. Also the average SNRs at all branches are not equal because the propagation paths of the receiver diversity branches are not identical, the receiver electronics is not perfect and can be unbalanced [6].

Considering the related works on MRC and EGC, a perfect carrier phase estimation of the incoming signal was mainly assumed $[4,5,7]$. However, only in $[8,9]$ the influence of the imperfect estimation of the received signal phase to the EGC system performance was discussed. In [8] the phase error influence on the bit error rate (BER) values in detecting digital binary phase-shift keying (BPSK) and quaternary phase-shift keying (QPSK) signals was discussed. The analysis was carried out under the assumption that the identical and statistically independent Rayleigh fading is present at receiver antennas. In [9] closed-form expressions were derived for the outage probability and the average BER in detecting BPSK and QPSK signals transmitted over correlated Nakagami- $m$ fading channels. The EGC technique with dual branches was observed in that paper.

In this paper, under the assumption of imperfect phase estimation, we compare the error performance of MRC and EGC diversity receivers with unbalanced branches and BPSK and QPSK modulations over correlated Weibull channels. The numerical and simulations results demonstrate the impact of the carrier phase error, as well as correlation and unbalance coefficient, on the MRC and EGC error performance.

The reminder of the paper is organized as follows. In Sect. 2, we consider channel and receiver model, and describe the analytical and simulations approach for BER performance determination. Section 3 provides numerical and simulations results with appropriate discussions, and Sect. 4 offers some concluding remarks.

\section{Performance Analysis}

\subsection{System Model}

The signal at the $i$-th receiver antenna can be written as

$$
s_{i}(t)=r_{i}(t) \exp \left(j \gamma_{i}(t)\right) A \exp \left(j\left(\omega_{0} t+\phi_{n}\right)\right)+n_{i}(t), \quad i=1,2,
$$

where $r_{i}(t)$ is the fading envelope, $\omega_{0}$ is the angular frequency, $\gamma_{i}(t)$ is the random phase shift which occurred during the signal transmission over a fading channel. The amplitude of useful signal is denoted with $A$ and it can be assumed, without loss of generality, that it is equal to one. With $\phi_{n}$ we denote the signal phase in which information about sent symbol is written. In the case of the BPSK signal $\phi_{n}$ has one of the following values: $\{0, \pi\}$, and in the case of the QPSK signal $\phi_{n}$ has one of the following values: $\{\pi / 4,3 \pi / 4,5 \pi / 4,7 \pi / 4\}$. The fading at each antenna is frequency nonselective, during one symbol it does not change, it is independent from symbol to symbol, but there is a correlation between fading on different antennas. The corresponding joint probability density function (PDF) of the fading envelopes (denoted by $r_{1}$ and $r_{2}$ ) at the first and second branch is Weibull [10] 


$$
\begin{aligned}
p_{r_{1}, r_{2}}\left(r_{1}, r_{2}\right)= & \frac{\alpha_{1} \alpha_{2}}{\Omega_{1} \Omega_{2}(1-\rho)} r_{1}^{\alpha_{1}-1} r_{2}^{\alpha_{2}-1} \exp \left(-\frac{r_{1}^{\alpha_{1}}}{\Omega_{1}(1-\rho)}\right) \\
& \times \exp \left(-\frac{r_{2}^{\alpha_{2}}}{\Omega_{2}(1-\rho)}\right) I_{0}\left(\frac{2 \sqrt{\rho} r_{1}^{\alpha_{1} / 2} r_{2}^{\alpha_{2} / 2}}{\sqrt{\Omega_{1} \Omega_{2}}(1-\rho)}\right), \quad r_{1}, r_{2} \geq 0,
\end{aligned}
$$

where $\rho$ is the correlation coefficient between two branches, $\alpha_{1}$ and $\alpha_{2}$ are the fading parameters in the first and second branch, respectively. The mathematical expectation is denoted by $\mathrm{E}\{$.$\} . It is worth \Omega_{1}=E\left\{r_{1}^{\alpha_{1}}\right\}, \Omega_{2}=E\left\{r_{2}^{\alpha_{2}}\right\}$. The modified Bessel function of the first kind and zero order is denoted by $\mathrm{I}_{0}($.$) [11, eq. (8.406)].$

The zero mean Gaussian noise with variance $\sigma_{i}^{2}$ at the $i$-th receiver branch is denoted with $n_{i}(t)(i=1,2)$. The standard deviation of this Gaussian noise is given by

$$
\sigma_{i}=\sqrt{\mathrm{E}\left\{r_{i}^{2}\right\} /\left[2 \log _{2} M 10^{\gamma_{b 1} / 10} \exp (-\delta(i-1))\right]}, \quad i=1,2,
$$

where $M$ is the number of phase levels, $\delta$ is the unbalancing coefficient (power decaying factor) and $\gamma_{b 1}$ is the average SNR per bit for the first receiver branch and is given in decibels.

After signal cophasing at all branches and multiplying by the estimated signal envelope at each branch (the received signal at the $i$-th branch is multiplied by $\left.r_{i}(t) \exp \left(-j\left(\omega_{0} t+\hat{\gamma}_{i}(t)\right)\right)\right)$, the resulting signal after MR combining is

$$
z_{M R C}(t)=\sum_{i=1}^{2}\left(A r_{i}^{2}(t) e^{j \phi_{n}} e^{j \varphi_{i}(t)}+r_{i}(t) n_{i}(t)\right)
$$

In the case of EG combining (the received signal at the $i$-th branch is multiplied by $\left.\exp \left(-j\left(\omega_{0} t+\hat{\gamma}_{i}(t)\right)\right)\right)$, the resulting signal after signal cophasing at all branches is

$$
z_{E G C}(t)=\sum_{i=1}^{2}\left(A r_{i}(t) e^{j \phi_{n}} e^{j \varphi_{i}(t)}+n_{i}(t)\right) .
$$

The difference between the received signal phase $\gamma_{i}(t)$ at the $i$-th receiver branch and the estimated phase $\hat{\gamma}_{i}(t)$ at that receiver branch is denoted with $\varphi_{i}(t)=\gamma_{i}(t)-\hat{\gamma}_{i}(t)$ in both cases. Assuming that the reference carrier phase is derived from unmodulated carrier using phase-locked loop (PLL) with a first order feedback filter, and if the Gaussian noise is the only one present in the PLL circuit, then the PDF of this phase error is given by $[8,9]$

$$
p_{\varphi_{i}}\left(\varphi_{i}\right)=\frac{1}{2 \pi} \frac{\exp \left(\zeta_{i} \cos \left(\varphi_{i}\right)\right)}{\mathrm{I}_{0}\left(\varsigma_{i}\right)}, \quad-\pi<\varphi_{i} \leq \pi, \quad i=1,2
$$

where $\zeta_{i}$ is the signal-to-noise ratio in the PLL circuit at the $i$-th receiver branch, which can be denoted through phase error variance $\sigma_{\varphi_{i}}^{2}[7,8]$

$$
\zeta_{i}=1 / \sigma_{\varphi_{i}}^{2}
$$




\subsection{Analytical Approach}

After analysis of the MRC receiver and mathematical manipulations it can be shown that the average BER of QPSK signal detection is given by

$$
\begin{aligned}
P_{e}= & 0.25 \iint_{r_{1}} \int_{r_{2}} \int_{\varphi_{1}}\left\{\operatorname{erfc}\left(\frac{\sum_{i=1}^{2} r_{i}^{2} \cos \left(\pi / 4-\varphi_{i}\right)}{\sqrt{2} \sigma_{M R C}}\right)\right. \\
& \left.+\operatorname{erfc}\left(\frac{\sum_{i=1}^{2} r_{i}^{2} \cos \left(\pi / 4+\varphi_{i}\right)}{\sqrt{2} \sigma_{M R C}}\right)\right\} \\
& \times p_{\varphi_{1}}\left(\varphi_{1}\right) p_{\varphi_{2}}\left(\varphi_{2}\right) p_{r_{1}, r_{2}}\left(r_{1}, r_{2}\right) d \varphi_{2} d \varphi_{1} d r_{2} d r_{1},
\end{aligned}
$$

In the case of EGC receiver, the average BER of QPSK signal detection is given by

$$
\begin{aligned}
P_{e}= & 0.25 \iint_{r_{1}} \int_{r_{2}} \int_{\varphi_{1}}\left\{\operatorname{erfc}\left(\frac{\sum_{\phi_{2}}^{2} r_{i} \cos \left(\pi / 4-\varphi_{i}\right)}{\sqrt{2} \sigma_{E G C}}\right)\right. \\
& \left.+\operatorname{erfc}\left(\frac{\sum_{i=1}^{2} r_{i} \cos \left(\pi / 4+\varphi_{i}\right)}{\sqrt{2} \sigma_{E G C}}\right)\right\} \\
& \times p_{\varphi_{1}}\left(\varphi_{1}\right) p_{\varphi_{2}}\left(\varphi_{2}\right) p_{r_{1}, r_{2}}\left(r_{1}, r_{2}\right) d \varphi_{2} d \varphi_{1} d r_{2} d r_{1} .
\end{aligned}
$$

The complementary error function is denoted by $\operatorname{erfc}().\left[11\right.$, eq. (7.1.2.)], $p_{\varphi_{1}}\left(\varphi_{1}\right)$ and $p_{\varphi_{2}}\left(\varphi_{2}\right)$ denote PDFs of the phase error at the first and second branch, respectively. The joint PDF of the fading envelopes at the first and second branch is marked as $p_{r_{1}, r_{2}}\left(r_{1}, r_{2}\right)$ and is given by (2). In the case of MRC receiver it is worth $\sigma_{M R C}=\sqrt{r_{1}^{2} \sigma_{1}^{2}+r_{2}^{2} \sigma_{2}^{2}}$, and in the case of EG combining it is worth $\sigma_{E G C}=\sqrt{\sigma_{1}^{2}+\sigma_{2}^{2}}$. Quite similarly one can obtain the expressions for BER computation in BPSK signal detection.

The numerical integration in (8)-(9) is performed by using Gaussian quadrature formulae with previously given precision accuracy. On the personal computer with a 2 GB RAM and a 2.4 GHz AMD Phenom processor running Windows XP, using Mathematica 6, a BER value of about $10^{-6}$ with 6 effective digits of precision, is computed within less than $200 \mathrm{~s}$.

\subsection{Simulation}

The computer simulation was performed using $\mathrm{C}^{++}$programming language. The information bits are uniformly generated and Gray mapped giving the information bearing phase $\phi_{n}$. The correlated Weibull fading envelopes $r_{1}(t)$ and $r_{2}(t)$ from (4) and (5) are generated by using the algorithm from [12]. For given value of phase noise standard deviation $\sigma_{\varphi i}$ the PLL $\mathrm{SNR} \zeta_{i}$ is calculated from (7), and the phase error $\varphi_{i}(t)$ with the Tikhonov PDF given by (6) is generated by the acceptance/rejection method [13, pp. 381-382]. The Gaussian noise $n_{i}(t)$ 


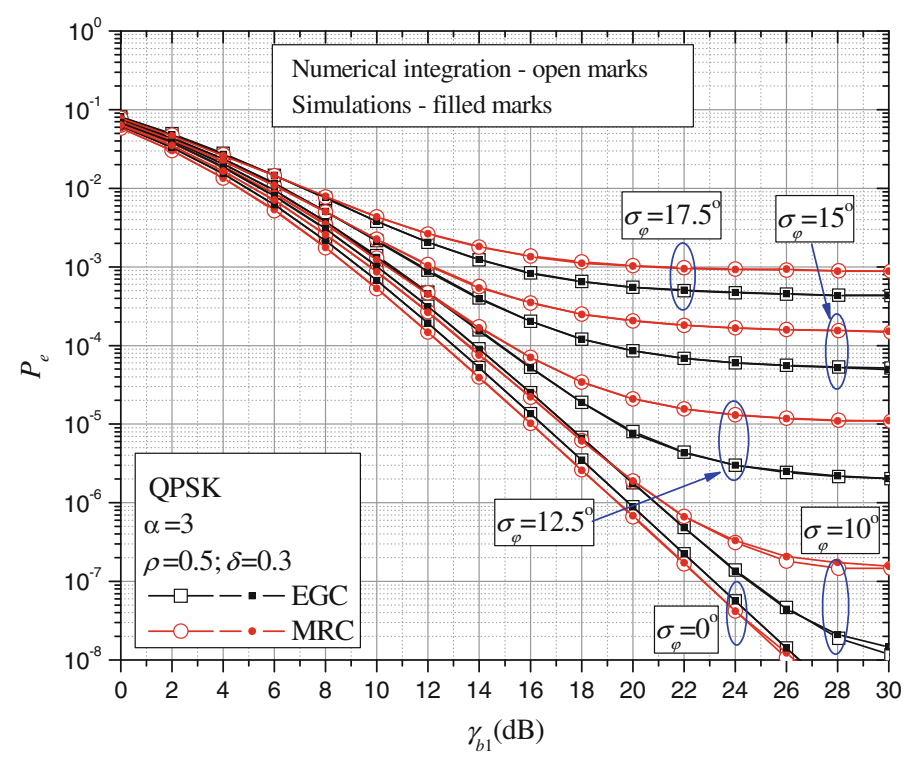

Fig. 1 MRC and EGC receiver performance for different values of phase noise standard deviation

from (4) and (5), with zero mean and standard deviation given by (3), is generated by using the Box-Muller method [13, p. 383-384]. The decision is made on the basis of the sampled values of $z_{M R C}(t)$ and $z_{E G C}(t)$, given by (4) and (5). The simulation results are obtained by using the Monte Carlo simulations [13, p. 686-703]. The BER values are estimated on the basis of $3 \cdot 10^{3}$ bit errors. In addition, a minimum number of bits used for evaluating any BER value is $10^{4}$, and maximum number of bits used in simulations is $2 \cdot 10^{9}$.

\section{Numerical and Simulation Results}

In this section, based on proposed analysis, numerical and simulations results are presented in order to illustrate EGC and MRC receiver degradations caused by simultaneous influences of carrier signal phase error, correlation of fading channels and receiver branches unbalance.

For the convenience of the results presentation and without loss of generality, the assumption is that in both receiver branches the phase noise standard deviations are same $\sigma_{\varphi 1}=$ $\sigma_{\varphi 2}=\sigma_{\varphi}$ and so are the fading parameters $\alpha_{1}=\alpha_{2}=\alpha$.

Figure 1 presents the average BER dependence on the average SNR per bit of the first branch, denoted by $\gamma_{b 1}$, for both MRC and EGC. In the case of perfect cophasing $\left(\sigma_{\varphi}=0^{0}\right)$ the BER values of MRC are lower than those of EGC, as it is expected. But when cophasing is not perfect it is interesting to note following: in domain of low values of the $\gamma_{b 1}$, the performance of MRC receiver is only slightly better, while EGC receiver shows significantly better characteristics for larger values of the $\gamma_{b 1}$. The irreducible error floor of EGC receiver is lower than that of MRC receiver.

In Figs. 2 and 3 the influence of the correlation coefficient, denoted by $\rho$, on the BER values in the case of EG and MR combining is shown, respectively. It can be observed that, for small values of $\gamma_{b 1}$, correlation unfavorable affects the system performance. For larger values of the $\gamma_{b 1}$, however, the BER floor appears but correlation positively influences the 


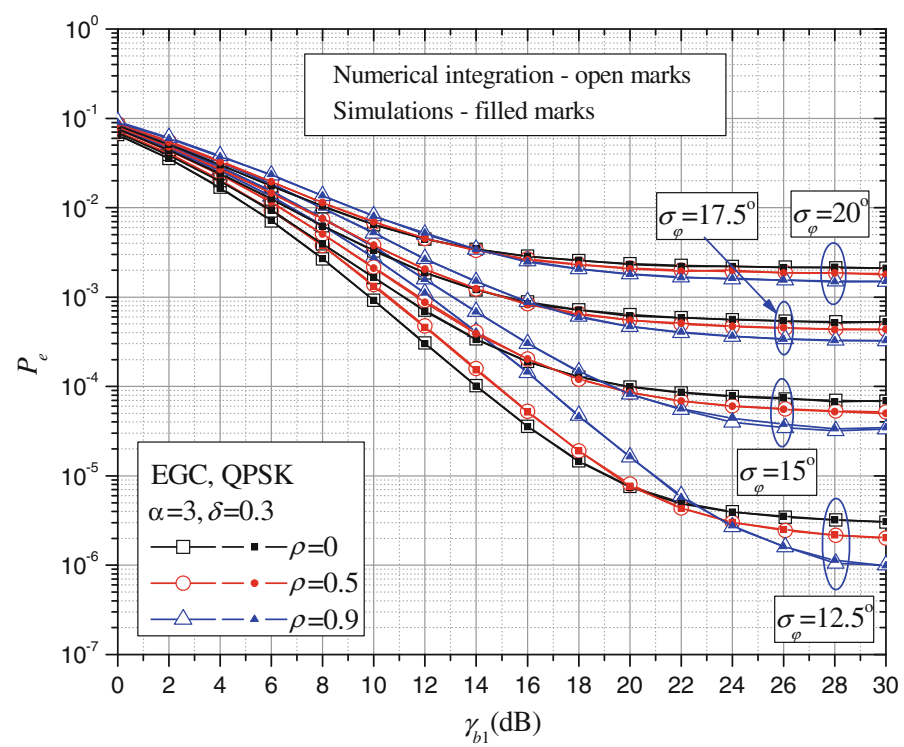

Fig. 2 EGC receiver performance for different values of correlation coefficient

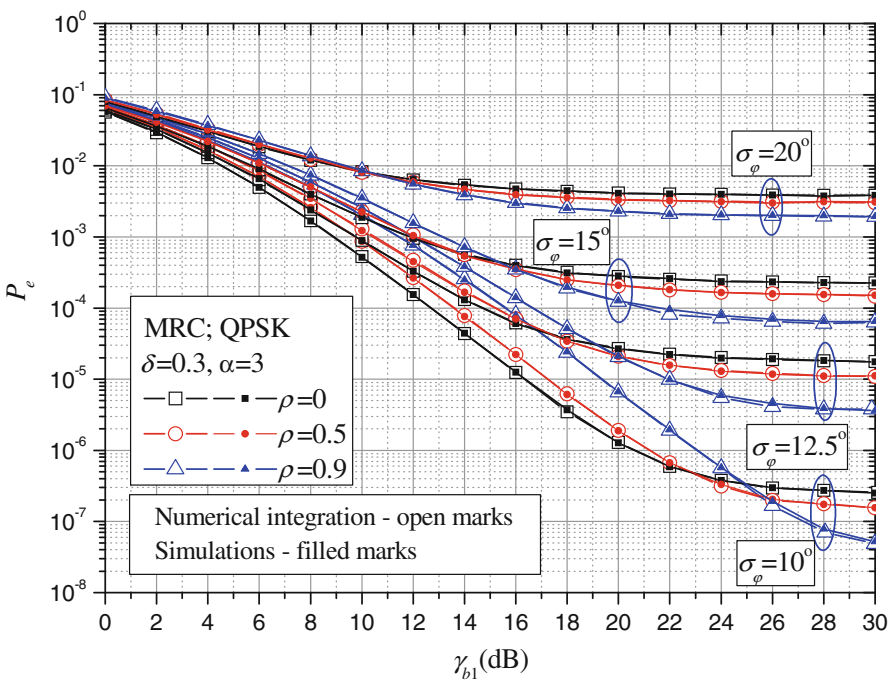

Fig. 3 MRC receiver performance for different values of correlation coefficient

system performance. The higher the value of $\rho$, the lower the value of the BER floor is. This effect is expressed both for EGC and MRC receivers. The similar effect was also examined in [9] for EGC receiver in correlated Nakagami- $m$ fading.

The influence of the branch unbalance on the EGC receiver performance for BPSK signaling is presented in Fig. 4. The coefficient of the branch unbalance, denoted by $\delta$, significantly affects the average BER values in the range of small and moderate values of the $\gamma_{b 1}$. For large 


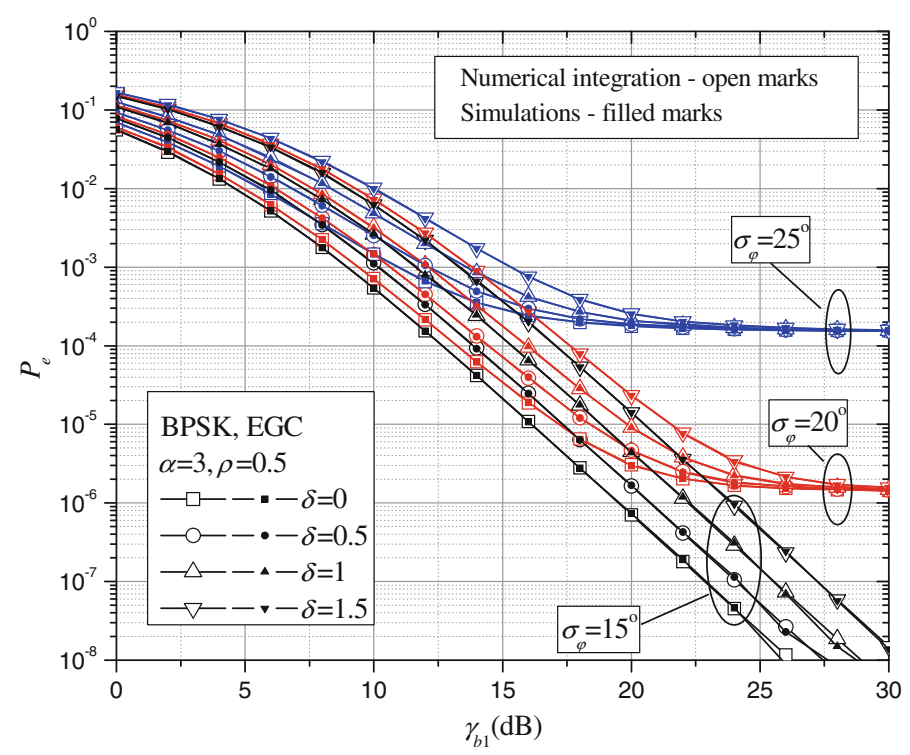

Fig. 4 EGC receiver performance for different values of unbalance coefficient

values of the $\gamma_{b 1}$, the BER floor appears and its value does not depend on the coefficient of branch unbalance.

\section{Conclusion}

In this paper, the performance of dual MRC and EGC receivers with unbalanced branches, operating over correlated Weibull fading channels, was studied. The formulae for average BER in detecting QPSK signals are obtained. The BER values were determined by performing numerical integration with a certain accuracy and confirmed by Monte Carlo simulations. The numerical and simulations results are in excellent agreement.

It was found that, in the presence of imperfect cophasing when carrier phase errors satisfy Tikhonov distribution, EGC has lower error floor than MRC. Similarly as in [9], it was shown that the higher value of correlation parameter between branches, the lower value of BER floor is in the case of both MRC and EGC. The results also illustrated that the unbalance parameter considerably influences the BER values in the range of low and moderate average SNR values, but does not affect the BER floor values that are dominantly influenced by the imperfect cophasing and the level of correlation.

\section{References}

1. Babich, F., \& Lombardi, G. (2000). Statistical analysis and characterization of the indoor propagation channel. IEEE Transactions on Communications, 48(3), 455-464.

2. Tzeremes, G., \& Christodoulou, C. G. (2002). Use of Weibull distribution for describing outdoor multipath fading. Antennas and Propagation Society International Symposium, 1, 232-235.

3. Cheng, J., Tellambura, C., \& Beaulieu, N. C. (2004). Performance of digital linear modulations on Weibull slow-fading channels. IEEE Transactions on Communications, 52(8), 1265-1268. 
4. Simon, M. K., \& Alouini, M. S. (2005). Digital communication over fading channels, 2nd ed. New York: Wiley.

5. Karagiannidis, G. K., Zogas, D. A., Sagias, N. C., Kotsopoulos, S. A., \& Tombras, G. S. (2005). Equalgain and maximal-ratio combining over Nonidentical Weibull fading channels. IEEE Transactions on Wireless Communications, 4(3), 841-846.

6. Dong, X., \& Beaulieu, N. C. (2002). Average level crossing rate and average fade duration of low-order maximal ratio diversity with unbalanced channels. IEEE Communications Letters, 6(4), 135-137.

7. Sagias, N. C., Karagiannidis, G. K., Mathiopoulos, P. T., \& Tsiftsis, T. A. (2006). On the performance analysis of equal-gain diversity receivers over generalized Gamma fading channels. IEEE Transactions on Wireless Communications, 5(10), 2967-2974.

8. Najib, M. A., \& Prabhu, V. K. (2000). Analysis of equal-gain diversity with partially coherent fading signals. IEEE Transactions on Vehicular Technology, 49(3), 783-791.

9. Sagias, N. C., \& Karagiannidis, G. K. (2005). Effects of carrier phase error on EGC receivers in correlated Nakagami-m fading. IEEE Communications Letters, 9(7), 580-582.

10. Sagias, N. C., \& Karagiannidis, G. K. (2005). Gaussian class multivariate Weibull distributions: theory and applications in fading channels. IEEE Transactions on Information Theory, 51(10), 3608-3619.

11. Gradsteyn, I. S., \& Ryzhik, I. M. (2000). Table of Integrals, Series, and Products, 6th ed. New York: Academic.

12. Matolak, D. W., Sen, I., \& Xiong, W. (2008). Generation of multivariate Weibull random variates. IET Communications, 2(4), 523-527.

13. Jeruchim, M. C., Balaban, P., \& Shanmugan, K. S. (2000). Simulation of communication systemsmodeling, methodology, and techniques. New York: Kluwer.

\section{Author Biographies}

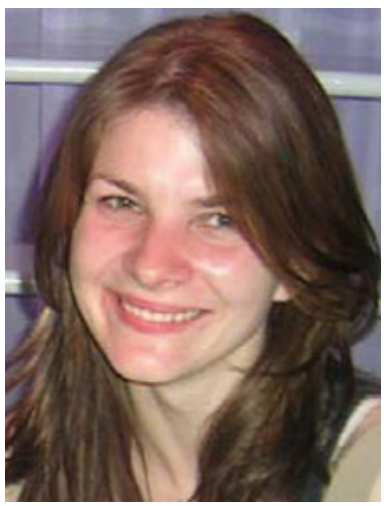

Bojana Z. Nikolic was born in Nis, Serbia in 1982. In 2007 she graduated from the Faculty of Electronic Engineering in Nis, major Telecommunications and enrolled Ph.D. studies on same faculty. She is a coauthor of the several papers, presented at national and international conferences. Currently, Ms. Nikolic is a Teaching Assistant at the Faculty of Electronic Engineering in Nis.

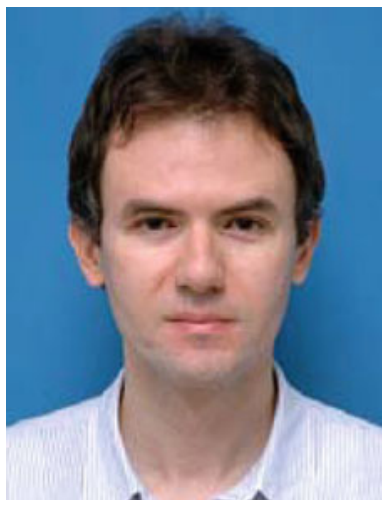

Goran T. Djordjevic was born in Nis, Serbia. He received his B.S., M.S., and Ph.D. degrees in Electrical Engineering from the Faculty of Electronic Engineering, University of Nis, Serbia, in 1996, 1999 and 2005 , respectively. His area of interest is communication theory and applications in satellite, wireless and optical communication systems. His current research interests include application of different modulation formats and error control codes in satellite fixed and mobile services, modeling and simulation of fading channels, synchronization problems. Currently he is an Assistant Professor at the Department of Telecommunications, Faculty of Electronic Engineering, University of Nis, Serbia. He teaches courses of Statistical Communication Theory, Modeling and Simulation of Communication Systems, Information Theory and Satellite Communications. Dr. Djordjevic is a recipient of the 2005 Best Young Scientists Paper Award at the 49th ETRAN Conference and 2007 Prof. Dr. Ilija Stojanovic Award for the contribution in the field of communications. 


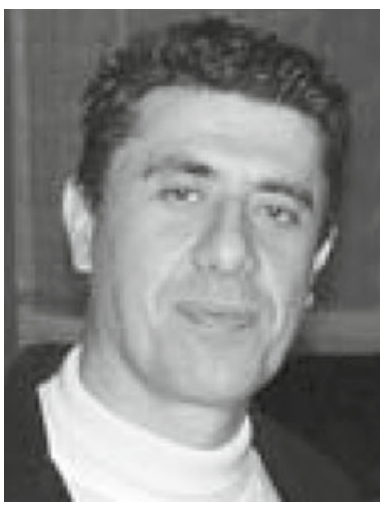

George K. Karagiannidis (M'97-SM'04) was born in Pithagorion, Samos Island, Greece. He received the University and Ph.D. degrees in electrical engineering from the University of Patras, Patras, Greece, in 1987 and 1999, respectively. From 2000 to 2004, he was a Senior Researcher at the Institute for Space Applications and Remote Sensing, National Observatory of Athens, Greece. In June 2004, he joined Aristotle University of Thessaloniki, Thessaloniki, Greece, where he is currently Associate Professor on Digital Communication Systems, in the Electrical and Computer Engineering Department. His current research interests include wireless communication theory, digital communications over fading channels, cooperative diversity systems, cognitive radio, satellite communications, and wireless optical communications. He is the author or coauthor of more than 90 technical papers published in scientific journals and presented at international conferences. He is also a coauthor of two chapters in books and a coauthor of the Greek edition of a book on mobile communications. He serves on the editorial board of the EURASIP JOURNAL ON WIRELESS COMMUNICATIONS AND NETWORKING. Dr. Karagiannidis has been a member of Technical Program Committees for several IEEE conferences. $\mathrm{He}$ is a member of the editorial boards of the IEEE TRANSACTIONS ON COMMUNICATIONS and the IEEE COMMUNICATIONS LETTERS. He is co-recipient of the Best Paper Award of the Wireless Communications Symposium (WCS) in IEEE International Conference on Communications (ICC' 07), Glasgow, U.K., June 2007. 